Adıyaman Üni. Sağlık Bilimleri Derg, 20119; 5(1);1246-1253

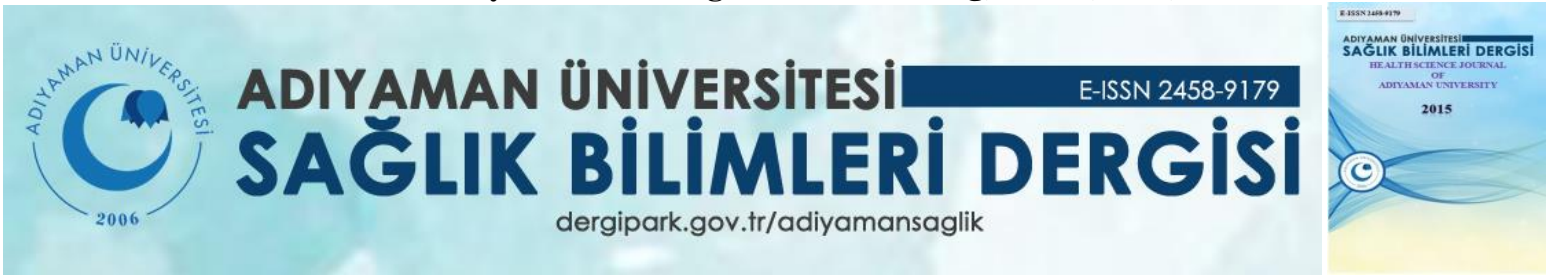

\title{
Araștırma/Research \\ Açık kalp cerrahisinde geleneksel yönteme alternatif sternum kapama tekniğimiz
}

Arda Aybars PALA ${ }^{1}$, Hasan İNER ${ }^{1}$, Murat Abdulhamit ERCISLI ${ }^{2}$

${ }^{1}$ Adıyaman Eğitim ve Araştırma Hastanesi, Kalp ve Damar Cerrahisi Kliniği, Adıyaman, Türkiye

${ }^{2}$ Adıyaman Üniversitesi Tıp Fakültesi, Kalp ve Damar Cerrahisi Ana Bilim Dalı, Adıyaman, Türkiye

$\ddot{O} z$

Giriş: Açık kalp cerrahisinde postoperatif süreçte görülen nadir fakat ciddi bir komplikasyon sternal dehisenstir. Sternal dehisensi önlemek için sternumun iyi kapatılması şarttır. Çelik tellerle sternumun birleştirilmesi konvansiyonel sternum kapama tekniği olarak kabul görmektedir. Biz bu çalışmada, kliniğimizde çelik teller ile uyguladığımız alternatif bir sternum kapama tekniğini ve sonuçlarını sunmayı amaçladık.

Yöntem: Kliniğimizde Ocak 2017 ile Ocak 2018 tarihleri arasında açık kalp cerrahisi uygulanmış 115 hastanın verileri retrospektif olarak değerlendirildi. Hastaların hepsinin sternum kapatılması uyguladığımız alternatif teknik ile yapıldı. Hastaların sternal dehisens için risk faktörleri ve postoperatif verileri değerlendirildi.

Bulgular: Postoperatif süreçte $\% 4.3$ oranında yüzeysel sternal yara yeri enfeksiyonu gelişti. Hiçbir hastada derin sternal enfeksiyon (mediastinit) ve sternal dehisens gelişmedi. Dört hastada sternal komplikasyona bağlı olmayan mortalite görüldü. 106 hastada (\%92.1) sorunsuz olarak sternum stabilitesi ve primer yara iyileşmesi sağlandı.

Sonuç: Uyguladığımız sternum kapama tekniğinin sternal komplikasyonları önlemede etkin ve kolay uygulanabilir bir teknik olduğu düşüncesindeyiz.

Anahtar kelimeler: Açık kalp cerrahisi, sternal dehisens, sternum kapama

\begin{tabular}{l}
\hline Yazışmadan Sorumlu Yazar \\
\hline Arda Aybars PALA \\
Adıyaman Eğitim ve Araştırma Hastanesi, Kalp \\
ve Damar Cerrahisi Kliniği, \\
Tel : +900532710 4587 \\
Email: ardaaybars@ @otmail.com \\
\hline
\end{tabular}

Doi:10.30569.adiyamansaglik.503388

\begin{tabular}{ll}
\hline Geliş Tarihi: & 26.01.2019 \\
\hline Kabul Tarihi: & $\mathbf{0 5 . 0 2 . 2 0 1 9}$ \\
\hline
\end{tabular}




\title{
Alternative sternum closure technique to traditional method in open heart surgery
}

\begin{abstract}
Objectives: Sternal dehiscence is a rare but serious complication seen in postoperative period in cardiac surgery. A good closure of the sternum is essential to prevent sternal dehiscence. Closure of sternum by steel wires is accepted as conventional sternal closure technique. In this study, we aimed to present an alternative sternal closure technique and its results with steel wires in our clinic.
\end{abstract}

Methods: The data of 115 patients who underwent open heart surgery between January 2017 and January 2018 in our clinic were evaluated retrospectively. Sternal closure of all patients was performed with our alternative technique. Risk factors for sternal dehiscence and postoperative data of patients were evaluated.

Results: In the postoperative period, superficial sternal wound infection occurred at a rate of $4.3 \%$. None of the patients developed deep sternal infection (mediastinitis) and sternal dehiscence. Four patients had mortality and were not related to sternal complications. Sternal stability and primary wound healing were achieved in 106 patients $(92.1 \%)$ without any problem.

Conclusion: We think that our sternal closure technique is an effective and easily applicable technique to prevent sternal complications.

Keywords: Open heart surgery, sternal dehiscence, sternal closure 


\section{Giriş:}

Kalbe ve mediastinal yapılara ulaşmak için günümüzde en çok kullanılan insizyon median sternotomidir (1). Postoperatif süreçte median sternotomiye bağlı nadir görülen fakat ciddi bir komplikasyon sternal dehisenstir. Sternal dehisens, göğüs ön duvarında ağrı, pulmoner disfonksiyon, yüzeysel ve derin sternum yara yeri enfeksiyonları gelişmesine sebep olup morbidite ve mortaliteyi arttırabilir. Sternal komplikasyonlar \%0.3-5 oranında görülmesine rağmen mortalite oranı \%14-47 olarak bildirilmiştir $(2,3)$.

Sternal dehissens genellikle postoperatif ilk günler ya da haftalarda oluşur. Bunu engellemek için iyi sternal sabitleme şarttır. Sternal dehisens gelişmesinde hasta özellikleri ve kemik yapısı kadar uygulanan cerrahi kapama tekniği de önemlidir (4). Günümüze kadar bu konuda birçok çalışma yapılmış, farklı materyaller ve tekniklerle sternum kapatılmıştır. Fakat en iyi materyal ve teknik konusunda görüş birliği sağlanamamaktadır. Median sternotomi sonrası sternumun çelik tellerle birleştirilmesi uygulama kolaylığı, maliyet ve etkinlik bakımından konvansiyonel sternum kapama yöntemi olarak kabul görmüştür $(5,6)$.

Biz bu çalışma ile kliniğimizde yine çelik teller ile fakat farklı bir teknik ile uyguladığımız sternum kapama prosedürünün postoperatif sonuçlarının değerlendirmesini sunmayı amaçladık.

\section{Gereç ve yöntemler:}

Ocak 2017 ile Ocak 2018 tarihleri arasında Adıyaman Eğitim ve Araştırma Hastanesi Kalp ve Damar Cerrahisi Kliniğinde açık kalp cerrahisi uygulanmış 115 hastanın verileri retrospektif olarak incelendi. Çalışmamız için kurum onayı alındı. Hastalar, demografik veriler ve sternal dehisens için risk faktörleri (vücut kitle indeksi, diyabetes mellitus, kronik böbrek yetmezliği, kronik obstruktif akciğer hastalığı, sigara kullanımı) bakımından değerlendirildi. Perioperatif verilerden kross klemp ve perfüzyon süreleri değerlendirildi. Postoperatif süreçte toplam drenaj, buna bağlı kullanılan kan ve kan ürünü miktarı ile kanama revizyonu nedeni ile reexplorasyon, yoğun bakım ve hastane yatış süreleri, yüzeysel ve derin yara yeri enfeksiyonu ile sternal dehisens gelişimi bakımından hastalar değerlendirildi.

Çalışmaya dahil edilen hastaların hepsinde sternum kapama monofilament paslanmaz çelik tel (no.5) ile yapıld1. Manubrium sterni proksimaline tekli tel transsternal uygulandi. Manubriosternal bileşke fiksasyonu için tel sırasıyla manubrium sterni distalinden transsternal, 
2. interkostal aralıklardan (IKA) parasternal ve tekrar manubrium sterni distalinden transsternal geçilerek ters figür-8 oluşturuldu. Sonra korpus sterni fiksasyonu için 2. İKA'a ekstra bir tekli tel daha parasternal uyguland1, 3. ve 4. iKA'lardan tel parasternal geçilerek ters figür-8 uygulandı ve son olarak korpus sterni distaline tekli tel 5. İKA'lardan parasternal geçilerek uygulandı. Tel dikişler orta hatta sıkıca bükülerek ve uçları sternal dokuya gömülerek sternum kapama tamamlandı (Şekil 1 ve Resim 1).

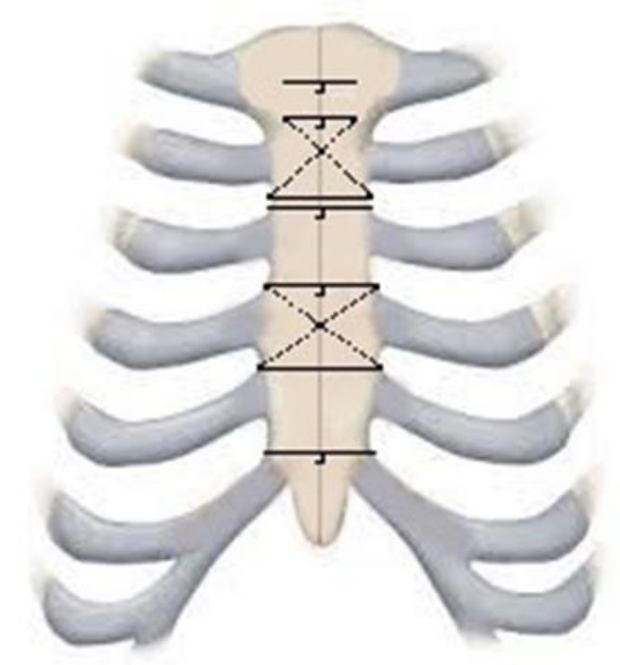

Şekil 1: Sternumun monofilament çelik teller ile kapatılmasının şematik olarak gösterilmesi

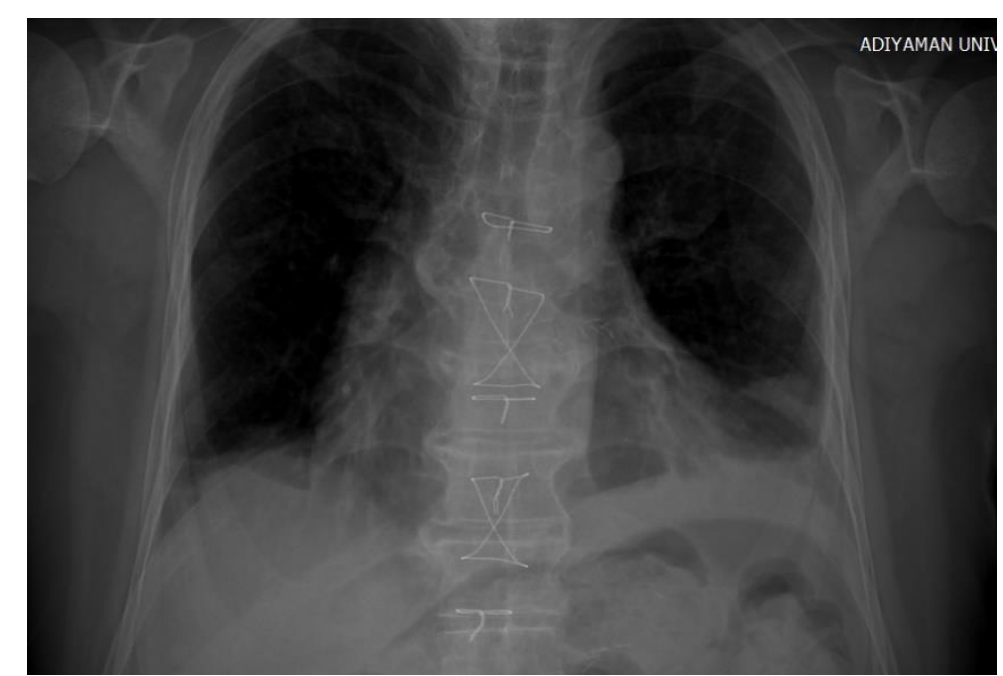

Resim 1: Gögü̈s radyogramında çelik tellerin görünümü 
Çalışmamızda demografik, preoperatif, perioperatif ve postoperatif verilerde sürekli değişkenler ortalama \pm standart sapma olarak, kategorik değişkenler ise hasta sayısı ve yüzde olarak ifade edildi.

\section{Bulgular:}

Demografik ve preoperatif veriler değerlendirildiğinde çalışmaya dâhil edilen 115 hastanın (82 erkek, 33 kadın) ortalama yaşı $63 \pm 8.7$ idi. Ortalama vücut kitle indeksi (VKİ) $27.2 \pm 4.9 \mathrm{~kg} / \mathrm{m}^{2}$ olarak bulundu. Hastaların \%59.1' inde diabetes mellitus, \%4.3' ünde kronik böbrek yetmezliği, \%33.9' unda kronik obstruktif akciğer hastalığı ve \%60.8' inde sigara kullanımı vard1 (Tablo 1).

Tablo 1: Demografik ve preoperatif veriler

\begin{tabular}{|c|c|c|c|}
\hline & Hasta Sayısı & $\%$ & $\begin{array}{c}\text { Ortalama } \pm \text { Standart } \\
\text { Sapma }\end{array}$ \\
\hline Yaş & & & $63 \pm 8.7$ \\
\hline Cinsiyet & & & \\
\hline Erkek & 82 & 71.3 & \\
\hline Kadın & 33 & 28.7 & \\
\hline VKİ $\left(\mathrm{kg} / \mathrm{m}^{2}\right)$ & & & $27.2 \pm 4.9$ \\
\hline $\mathrm{DM}$ & 68 & 59.1 & \\
\hline KBY & 5 & 4.3 & \\
\hline KOAH & 39 & 33.9 & \\
\hline Sigara kullanımı & 70 & 60.8 & \\
\hline
\end{tabular}

VKİ: Vücut Kitle İndeksi; DM: Diyabetes Mellitus; KBY: Kronik Böbrek Yetmezliği; KOAH: Kronik Obstruktif Akciğer Hastalığ

Uygulanan cerrahi prosedürler değerlendirildiğinde 90 hasta ve \%78.2 oran ile en fazla koroner arter baypas greft (KABG) cerrahisi uygulandığı görüldü (Tablo 2). KABG cerrahisi yapılan hastalar değerlendirildiğinde \%84.4 oranında sol internal mammarian arterin greft olarak kullanıldığ 1 saptand1.

Tablo 2: Cerrahi prosedürler

\begin{tabular}{l|c|c}
\hline & Hasta Sayisı & \% \\
\hline KABG & 90 & 78.2 \\
\hline MVR & 13 & 11.3 \\
\hline AVR & 5 & 4.3 \\
\hline KABG + MVR & 2 & 1.7 \\
\hline KABG + AVR & 2 & 1.7 \\
\hline AAR & 3 & 2.6 \\
\hline
\end{tabular}

KABG: Koroner Arter Baypas Greft; MVR: Mitral Kapak Replasmanı; AVR: Aort Kapak Replasmanı; AAR: Asendan Aort Replasmanı 
Perioperatif verilerde kross klemp süresi ortalama $71.88 \pm 20.75 \mathrm{dk}$ ve total perfüzyon süresi ortalama $116.86 \pm 29.54 \mathrm{dk}$ olarak bulundu. Postoperatif verilerde toraks ve mediasten drenlerinden toplam drenaj ortalama $589.71 \pm 172.94 \mathrm{ml}$ olarak bulundu ve buna bağlı olarak kullanılan kan ve kan ürünü sayısı ortalama $4 \pm 3.4$ Ü idi. 4 hastaya kanama revizyonu nedeni ile re-explorasyon uygulandı. Hastaların yoğun bakım yatış süreleri ortalama $2.6 \pm 1.03$ gün ve hastane yatış süreleri ortalama $8.17 \pm 2.75$ gün olarak bulundu. Hastaların hiçbirinde derin sternal enfeksiyon (mediastinit) ve sternal dehisens gelişmedi, 5 hastada (\%4.3) yüzeysel sternal yara yeri enfeksiyonu gelişti (Tablo 3). 4 hastada (\%3.4) mortalite görüldü. 106 hastada (\%92.1) sorunsuz olarak sternum stabilitesi ve primer yara iyileşmesi sağlandı.

Tablo 3: Perioperatif ve postoperatif veriler

\begin{tabular}{l|c|c|c}
\hline & Hasta Sayıs & $\%$ & $\begin{array}{c}\text { Ortalama } \pm \text { Standart } \\
\text { Sapma }\end{array}$ \\
\hline Kross klemp süresi (dk) & & & $71.88 \pm 20.75$ \\
\hline Perfüzyon süresi (dk) & & & $589.71 \pm 172.94$ \\
\hline Toplam drenaj (ml) & & 3.4 & $4 \pm 3.4$ \\
\hline $\begin{array}{l}\text { Kan ve kan ürünü } \\
\text { transfüzyonu (ünite) }\end{array}$ & 4 & & \\
\hline $\begin{array}{l}\text { Kanama revizyonu nedeni } \\
\text { ile re-explorasyon }\end{array}$ & & & $2.6 \pm 1.03$ \\
\hline $\begin{array}{l}\text { Yoğun bakım yatı̧ süresi } \\
\text { (gün) }\end{array}$ & & 4.3 & $8.17 \pm 2.75$ \\
\hline Hastane yatış süresi (gün) & 5 & 0 & \\
\hline $\begin{array}{l}\text { Yüzeysel yara yeri } \\
\text { enfeksiyonları }\end{array}$ & 0 & 0 & \\
\hline $\begin{array}{l}\text { Derin enfeksiyonlar } \\
\text { (mediastinit) }\end{array}$ & 0 & & \\
\hline Sternal dehisens & & & \\
\hline
\end{tabular}

\section{Tartışma:}

Median sternotomi 1950'li yıllarda kardiyak cerrahide kullanılmaya başlanmış ve günümüzde halen kalp cerrahisinde en sık kullanılan kesidir. Sternumun kapatılmasında iyi bir sternal sabitleme için paslanmaz çelik tel, sternal kablo, titanyum plak, talon sistemi, nitinol klips, kryptone kemik çimentosu, polidioksanon (pds) sütür ile sternal kapama gibi birçok farklı yöntem uygulanmış, bunlar üzerine birçok biyomekanik test ve klinik çalışmalar yapılmıştır. Maliyet, uygulama kolaylı̆̆ı, tekrar cerrahi müdahale gerektiğinde rahat çıkartılabilirlik ve etkinlik bakımından değerlendirildiğinde çelik teller öne çıkmaktadır. Median sternotominin ilk 
uygulandığı günden bu yana sternum kapatılmasında en yaygın teknik paslanmaz çelik tellerin kullanımıdır $(1,5,6)$.

Sternal dehisens kardiyak cerrahide \%0,5-5 oranında görülen ciddi bir sternal komplikasyondur. Sternal dehisens için preoperatif risk faktörleri; obezite, diyabetes mellitus, kronik böbrek yetmezliği, kronik obstruktif akciğer hastalığı, sigara kullanımı, kronik öksürük, osteoporoz ve reoperasyondur (3). Obezite major risk faktörü olarak kabul edilmektedir (7). Molina ve ark. (2004) tarafından yapılan bir çalışmada, VKİ 30' un üzerinde (obez) olan 1253 hastada sternal dehisens gelişme oranı \%6.46, VKİ 30' un altında olan 1905 hastada sternal dehisens gelişme oranı \%1.6 olduğu belirtilmiştir (8). Bizim çalışmamızdaki 115 hastanın ortalama VKİ $27.2 \pm 4.9 \mathrm{~kg} / \mathrm{m}^{2}$ (fazla kilolu, pre-obez) olarak bulunmuştur ve sternal dehisens gelişen hasta olmamıştır.

Perioperatif risk faktörleri arasında uzamış kardiyopulmoner baypas süresi, bilateral internal mammarian arter kullanımı ve aşırı bone wax kullanımı sayılabilir. Postoperatif süreçte ise kan ve kan ürünü transfüzyonları, re-explorasyon, uzamış yoğun bakım yatış süresi sternal dehisens için risk faktörü olarak bildirilmiştir (3). Açık kalp cerrahisi uygulanan 2760 hastalık bir çalışmada sternal dehisens oranı \%1.6 olarak rapor edilmiş ve bu hasta grubunda kanamaya bağlı re-explorasyon $(\mathrm{p}<0.05)$, uzamış kardiyopulmoner baypas süresi ve yoğun bakım yatış süresi $(\mathrm{p}<0.001)$ anlamlı olarak fazla bulunmuştur $(9)$. Bizim çalışmamızdaki hasta grubunda total perfüzyon süresi ortalama $116.86 \pm 29.54 \mathrm{dk}$, kanama revizyonu nedeni ile re-explorasyon uygulanan hasta sayısı 4 (\%3.4), yoğun bakım yatış süreleri ortalama $2.6 \pm 1.03$ gün olarak bulunmuştur.

Median sternotomi sonrası gelişen enfeksiyonlar yüzeysel sternal yara yeri enfeksiyonları ve derin sternal enfeksiyonlar (mediastinit) olarak iki gruba ayrılır (10). Derin sternal enfeksiyonların çoğunlukla sternal dehisens sonrasında geliştiği ve yüzeysel sternal yara yeri enfeksiyonlarının uygun tedavi olmazsa derin sternal enfeksiyonlara yol açıp morbidite ve mortaliteyi arttırdığı bilinmektedir. Kardiyak cerrahi sonrası sternum yara yeri enfeksiyonlarının \%0.25-5 oranında görüldüğü bildirilmiştir $(11,12)$. Bizim çalışmamızda, hastalarda derin sternal enfeksiyon gelişmediği ve 5 hastada (\%4.3) yüzeysel sternal yara yeri enfeksiyonu geliştiği görülmekle birlikte; uygun yara bakımı ve antibiyoterapi sonrası bu 5 hastada tam iyileşme sağlanmıştır. 
Sternal kapamada konvansiyonel yöntem olan çelik tellerle yapılan kapamalarda oluşabilecek komplikasyonları önlemek için tellerin farklı bağlandığı farklı teknikler yıllar içinde gelişmiştir. En sık kullanılan teknikler tekli tel ve figür-8 tekniğidir. Bu teknikleri karşılaştıran birçok çalışma yapılmıştır. Losanoff ve ark. (13) tarafindan domuz sternumu üzerinde yapılan bir çalışmada, parasternal tekli tel ve parasternal figür-8 tel teknikleri karşılaştırılmış ve parasternal tekli tel tekniğinde sternal dehisans gelişmesi için daha yüksek bir kuvvet gerektiği bildirilmiştir. Khasati ve ark. (14) tarafından yayınlanan meta-analizde, figür-8 tel tekniğinin tekli tel tekniğine üstünlüğü olmadığı ve telin transsternal ya da parasternal yerleştirilmesinin sternal stabiliteyi arttırmada daha önemli bir faktör olabileceği bildirilmiştir. Tekümit ve ark. (15) tarafından yapılan başka bir çalışmada, tekli tel veya figür- 8 tel tekniği ile tellenmiş 6211 kardiyak cerrahi hastası değerlendirilmiş, sternal dehisens oranı \%1.44 olarak bulunmuş ve her iki teknik arasında sternal dehisens gelişimi ve sternal dehisens gelişimi sonrası postoperatif seyir ve mortalite açısından anlamlı fark saptanmamıştır. Yapılan bunca çalışmaya rağmen hangi tekniğin sternum stabilitesi için daha etkin olduğu konusunda görüş birliği yoktur.

Biz bu çalışmamızda konvansiyonel çelik tellerle, figür-8 tekniğini modifiye ederek (ters figür8) ve tekli tel tekniği ile birleştirerek uyguladığımız teknikle sternum kapamasını gerçekleştirdiğimiz 115 hastanın verilerini değerlendirdik. Bu teknikte, manubriosternal bileşke fiksasyonunu arttırmak amacıyla çelik tel ile ters figür-8 uygulanmış ve sternum korpus fiksasyonu arttırarak sternum gevşemesini önlemek için 2. iKA'lara ekstra olarak tekli tel parasternal uygulanmıştır. Maliyet düşüklüğü, uygulama kolaylığı ve en önemlisi; hiçbir hastamızda derin sternal enfeksiyon (mediastinit) ve sternal dehisens gelişmediği için bu tekniğin alternatif bir teknik olarak sternum kapamasında güvenle kullanılabileceği düşüncesindeyiz. Bizim çalışmamızın limitasyonları arasında rölatif olarak hasta sayısının azlığı ve çalışmanın retrospektif planlanmış olması sayılabilir. Elbette konunun daha iyi irdelenebilmesi açısından prospektif ve randomize kontrollü çalışmalara ihtiyaç vardır. 


\section{Kaynaklar:}

1. Casha AR, Yang L, Kay PH, Saleh M, Cooper GJ. A biomechanical study of median sternotomy closure techniques. Eur J Cardiothorac Surg. 1999;15(3):365-9.

2. Robicsek F, Fokin A, Cook J, Bhatia D. Sternal instability after midline sternotomy. Thorac Cardiovasc Surg. 2000;48(1):1-8.

3. Losanoff JE, Richman BW, Jones JW. Disruption and infection of median sternotomy: a comprehensive review. Eur J Cardiothorac Surg. 2002;21(5):831-9.

4. Cohen DJ, Griffin LV. A biomechanical comparison of three sternotomy closure techniques. Ann Thorac Surg. 2002;73(2):563-8.

5. Kamiya H, Al-Maisary SS, Akhyari P, Ruhparwar A, Kallenbach K, Lichtenberg A et al. The number of wires for sternal closure has a significant influence on sternal complications in high-risk patients. Interact CardioVasc Thorac Surg. 2012;15:665-70.

6. Alhalawani AM, Towler MR. A review of sternal closure techniques. J Biomater Appl. 2013;28(4):483-97.

7. Kiessling AH, Isgro F, Weisse U, Möltner A, Saggau W, Boldt J. Advanced sternal closure to prevent dehiscence in obese patients. Ann Thorac Surg. 2005;80(4):1537-9.

8. Molina JE, Lew RS, Hyland KJ. Postoperative sternal dehiscence in obese patients: incidence and prevention. Ann Thorac Surg. 2004;78(3):912-7.

9. Bryan AJ, Lamarra M, Angelini GD, West RR, Breckenridge IM. Median sternotomy wound dehiscence: a retrospective case control study of risk factors and outcome. J R Coll Surg Edinb. 1992;37(5):305-8.

10. Lazar HL, Salm TV, Engelman R, Orgill D, Gordon S. Prevention and management of sternal wound infections. J Thorac Cardiovasc Surg. 2016;152:962-72.

11. Singh K, Anderson E, Harper JG. Overview and management of sternal wound infection. Semin Plast Surg. $2011 ; 25(1): 25-33$.

12. Dunne B, Murphy M, Skiba R, Wang X, Ho K, Larbalestier R et al. Sternal cables are not superior to traditional sternal wiring for preventing deep sternal wound infection. Interact Cardiovasc Thorac Surg. 2016;22:594-8.

13. Losanoff JE, Foerst JR, Huff H, Richman BW, Collier AD, Hsieh FH, et al. Biomechanical porcine model of median sternotomy closure. J Surg Res. 2002;107(1):108-12.

14. Khasati N, Sivaprakasam R, Dunning J. Is the figure-of-eight superior to the simple wire technique for closure of the sternum? Interact Cardiovasc Thorac Surg. 2004;3(1):191-4.

15. Tekümit H, Cenal AR, Tataroğlu C, Uzun K, Akinci E. Comparison of figure-of-eight and simple wire sternal closure techniques in patients with non-microbial sternal dehiscence. Anadolu Kardiyol Derg. 2009;9(5):4116. 